\title{
CDISC SDTM Coronary Artery Disease Presentation Terminology
}

National Cancer Institute

\section{Source}

National Cancer Institute. CDISC SDTM Coronary Artery Disease Presentation

Terminology. NCl Thesaurus. Code C101843.

Terminology associated with the coronary artery disease presentation codelist of the Clinical Data Interchange Standards Consortium (CDISC) Study Data Tabulation Model (SDTM). 